\title{
DESEMPENHO DE OVINOS E RESPOSTAS DE PASTAGENS DE TIFTON 85 (Cynodon spp.) SOB LOTAÇÃO CONTÍNUA
}

\author{
Roberta Aparecida Carnevalli ${ }^{1,4}$; Sila Carneiro da Silva $a^{2 *}$; Jaílson Lara Fagundes ${ }^{1,4}$; André Fischer \\ Sbrissia',3; Carlos Augusto Brandão de Carvalho, ${ }^{1,3}$; Luiz Felipe de Moura Pinto,4; Carlos \\ Guilherme Silveira Pedreira² \\ ${ }^{1}$ Pós-Graduandos do Depto. de Produção Animal - USP/ESALQ. \\ ${ }^{2}$ Depto. de Produção Animal - USP/ESALQ, C.P. 9 - CEP: 13418-900 - Piracicaba, SP. \\ ${ }^{3}$ Bolsista FAPESP. \\ ${ }^{4}$ Bolsista CAPES. \\ *Autor correspondente <scdsilva@carpa.ciagri.usp.br>
}

RESUMO: A utilização de pastagem como principal fonte de alimento para ruminantes vem crescendo nos últimos tempos devido a seu baixo custo de produção. Nesse sentido, plantas forrageiras que apresentem bom desempenho quando submetidas a pastejo e possuam flexibilidade de uso têm despertado grande interesse. Nesse contexto, foram avaliados o desempenho de ovinos e as características de pastagens de Tifton 85. Os pastos foram mantidos sob quatro intensidades de pastejo (tratamentos) correspondentes às alturas de 50, 100, 150 e $200 \mathrm{~mm}$ por ovinos em regime de lotação contínua com carga variável no período de agosto/1998 a abril/1999. Os dados foram reunidos por estação do ano (primavera e verão). Os tratamentos foram alocados às unidades experimentais aleatoriamente num delineamento de blocos completos com quatro repetições. Cada unidade experimental $\left(400 \mathrm{~m}^{2}\right)$ foi pastejada por, no mínimo, dois cordeiros marcadores de ganho de peso para estimar a resposta animal, com três gaiolas de exclusão para determinar o acúmulo de forragem. As respostas avaliadas foram: proteína bruta (PB), fibra em detergente neutro, fibra em detergente ácido, lignina, digestibilidade 'in vitro' da matéria orgânica (DIVMO) e composição morfológica da forragem como pastejada e a proporção de folhas, pseudohastes e material morto nos pastos, densidade de forragem, taxa de acúmulo de forragem, capacidade de carga animal, disponibilidade de forragem, ganho de peso por animal e por hectare e estimativas de consumo. As diferenças observadas em valor nutritivo foram maiores para os pastos de $50 \mathrm{~mm}$, os quais apresentaram os maiores valores de PB e DIVMO. O desempenho animal foi mais dependente da capacidade de carga animal e da disponibilidade de forragem nos pastos mais altos (150 e $200 \mathrm{~mm}$ ) apresentando os melhores desempenhos. Tanto desempenho animal quanto produção de forragem aumentaram no verão, como consequência do aumento das taxas de acúmulo de matéria seca. Palavras-chave: valor nutritivo, produção de forragem, composição morfológica, simulação de pastejo

\section{PASTURE AND SHEEP PERFORMANCE ON TIFTON 85 (Cynodon spp.) PASTURES UNDER CONTINUOUS STOCKING}

\begin{abstract}
The use of pastures for feeding ruminants is increasing in recent years due to its low cost comparatively to other sources of feed. In that sense, forage plants with good performance under grazing and presenting high flexibility of utilisation have raised a great deal of interest. In this context, pasture and animal performance were evaluated on Cynodon spp. (cultivar Tifton 85) swards kept in four steady state conditions (50, 100, 150 and $200 \mathrm{~mm}$ sward surface heights - SSH) with sheep grazing under continuous stocking and variable stocking rate from august/ 1998 to april/1999. Treatments corresponded to the SSH and were allocated to experimental units according to a complete randomised block design with four replications. Individual experimental units (400 $\mathrm{m}^{2}$ each) were grazed, at least, by two lambs to estimate animal responses, with three exclosure cages for herbage accumulation measurements. Experimental responses evaluated were: crude protein (CP), neutral detergent fiber, acid detergent fiber and lignin, and in vitro organic matter digestibility (IVOMD). In addition, morphological composition of the "grazed" forage, proportion of leaf, stem and dead material in swards, sward bulk density, herbage accumulation rate, carrying capacity, herbage allowance, liveweight gain per animal and per hectare were estimated, along with herbage intake. Despite the differences observed in the overall nutritive value $(P<0.05)$, where short swards $(50 \mathrm{~mm})$ had the highest values for CP and IVOMD, animal performance was more dependent on carrying capacity and herbage allowance, with animals performing better on longer swards (150 and $200 \mathrm{~mm}$ ). Animal performance and pasture production increased during summer as a consequence of the increased herbage accumulation rates during this period. Key words: nutritive value, herbage production, morphological composition, handing plucking
\end{abstract}

\section{INTRODUÇÃO}

O desempenho animal normalmente aumenta a taxas decrescentes até atingir um valor máximo à medida que a disponibilidade de forragem aumenta (Hodgson, 1984). O consumo de forragem por área é o produto do consumo por animal e o número de animais por unidade de área. O consumo de forragem por unidade de área, por 
sua vez, é igual ao acúmulo líquido de forragem e determina a eficiência de utilização da forragem em situações onde os pastos são mantidos em condições de estrutura constante sob lotação contínua. Uma alta taxa de consumo por unidade de área poderia ser o ideal se não fosse o conflito gerado entre as eficiências nas diferentes fases do processo de produção (Hodgson, 1990). A eficiência de conversão da forragem ingerida em produto animal aumenta progressivamente quando 0 consumo por animal aumenta. A eficiência de utilização da forragem, por outro lado, aumenta quando o consumo por unidade de área aumenta. O consumo por animal e por unidade de área são inversamente relacionados e isso significa que as eficiências do processo de utilização e conversão são também inversamente relacionadas (Hodgson, 1990).

Sob lotação contínua, a altura de pastejo é negativamente relacionada com as taxas de lotação, por causa dos efeitos combinados de menor eficiência de utilização e maior consumo por animal sob pastejo leniente (Hodgson, 1990). O consumo por animal e desempenho animal individual diminuem progressivamente quando a taxa de lotação aumenta. Isso sugere que o consumo é mais sensível ao declínio em disponibilidade de forragem do que ao declínio em valor nutritivo (Hodgson, 1990). O consumo normalmente se aproxima de um máximo em níveis de disponibilidade 3 a 4 vezes superiores à ingestão de matéria seca, mas apresenta redução acentuada quando a disponibilidade é inferior a duas vezes o consumo (Hodgson, 1984).

A disponibilidade de forragem é importante fator no consumo, porém a habilidade física do animal em colher a forragem e os efeitos da estrutura do pasto no comportamento ingestivo podem ser determinantes no controle da ingestão (Penning et al., 1991). Parsons et al. (1983) descreveram como pastos respondem ao pastejo, e mostraram que aumentando a intensidade de pastejo aumenta-se a proporção de folhas pastejadas, provocando uma redução na área foliar, fotossíntese e taxa bruta de produção de tecidos. A taxa bruta de produção de tecidos é restrita a um máximo em pastos mantidos com altos índices de área foliar, porém a quantidade colhida é pequena. Em baixo índice de área foliar, a reduzida produção de tecidos é determinada pelos baixos requerimentos de respiração e crescimento radicular e por um decréscimo na proporção de forragem não colhida, razão pela qual a quantidade de forragem colhida por hectare aumenta. Assim, a produção de forragem por área, sob lotação contínua, é maior em situações de índice de área foliar baixo a intermediário, obtido pelas altas taxas de lotação.

O principal problema do manejador de pasto é conciliar uma taxa de lotação que resulte em alto desempenho por animal com uma que resulte em melhor desempenho por unidade de área. Esta combinação não é facilmente encontrada em sistemas de produção nos quais o desempenho animal diminui constantemente com o aumento nas taxas de lotação, uma vez que nestas circunstâncias não é sensato fixar taxas de lotação restritas ao ponto de máximo desempenho por animal (Hodgson, 1984).

O objetivo deste estudo foi detalhar o conhecimento de como o desempenho animal e características do pasto respondem a mudanças na altura do pastejo (50, 100, 150 e 200 mm) em Cynodon spp. cv. Tifton 85.

\section{MATERIAL E MÉTODOS}

\section{Área experimental}

A espécie forrageira utilizada foi Cynodon spp. cultivar Tifton 85. O ensaio foi conduzido na Unidade Experimental de Plantas Forrageiras (UEPF) em área do Departamento de Produção Animal - Setor Ruminantes da Escola Superior de Agricultura "Luiz de Queiroz" da Universidade de São Paulo, em Piracicaba, São Paulo $\left(22^{\circ}\right.$ 42'30". S; 47 38'00". W; 546 m alt.). O solo da região é caracterizado como Terra Roxa Estruturada eutrófica, com horizonte A moderado e de textura argilosa/ muito argilosa. Por ocasião da instalação do experimento, a análise de solo revelava $\mathrm{pH}\left(\mathrm{CaCl}_{2}\right)=5,4 ; \mathrm{MO}\left(\mathrm{g} \mathrm{kg}^{-1}\right)=$ 37,$0 ; \mathrm{P}\left(\mathrm{mg} \mathrm{dm}^{-3}\right)=99 ; \mathrm{K}\left(\mathrm{cmol}_{\mathrm{c}} \mathrm{dm}^{-3}\right)=0,46 ; \mathrm{Ca}\left(\mathrm{cmol} \mathrm{dm}^{-3}\right)$ = 7,50; $\mathrm{Mg}\left(\mathrm{cmol}_{\mathrm{c}} \mathrm{dm}^{-3}\right)=2,60 ; \mathrm{H}+\mathrm{Al}\left(\mathrm{cmol}_{\mathrm{c}} \mathrm{dm}^{-3}\right)=3,00$; SB $\left(\mathrm{cmol}_{\mathrm{c}} \mathrm{dm}^{-3}\right)=10,60 ; \mathrm{T}\left(\mathrm{cmol}_{\mathrm{c}} \mathrm{dm}^{-3}\right)=13,60 \mathrm{e} \mathrm{V}(\%)=$ $78 \%$. Não foram realizadas adubações de correção devido à alta fertilidade do solo. Durante a condução do experimento em 1998 foram realizadas 6 adubações nitrogenadas com sulfato de amônio $\left(40 \mathrm{~kg} \mathrm{~N} \mathrm{ha}^{-1} \mathrm{em} 8\right.$ de

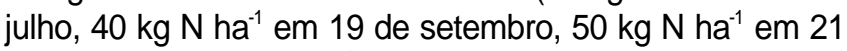
de outubro, $25 \mathrm{~kg} \mathrm{~N}$ ha $^{-1}$ em 7 de dezembro, $50 \mathrm{~kg} \mathrm{~N} \mathrm{ha}^{-1}$ em 23 de fevereiro, $75 \mathrm{~kg} \mathrm{~N}^{-1}{ }^{-1}$ em 20 de março).

$\mathrm{O}$ ensaio seguiu um delineamento em blocos completos casualizados com quatro repetições. Os tratamentos corresponderam a quatro condições de pasto caracterizadas pelas alturas de 50, 100, 150 e $200 \mathrm{~mm}$ mantidas relativamente "constantes" durante todo o período experimental através de pastejo por ovinos em regime de lotação contínua e taxa de lotação variável. Assim, o ensaio apresentou um total de 16 unidades experimentais (4 blocos $\times 4$ unidades/ bloco) com uma área média de $400 \mathrm{~m}^{2}$ cada. Os pastos atingiram as alturas dos tratamentos e passaram a ser controlados pelos animais a partir de junho de 1998. Em junho foram iniciadas as avaliações pré-experimentais e o período experimental foi de agosto de 1998 a abril de 1999. Durante o período experimental a temperatura média máxima diária foi de $31,4{ }^{\circ} \mathrm{C}$ e a mínima de $14,4{ }^{\circ} \mathrm{C}$. A precipitação total foi de $1290 \mathrm{~mm}$ e a umidade relativa do ar variou entre 64,8 e $90,0 \%$. Os dados climáticos do período experimental estão apresentados na TABELA 1.

\section{Monitoramento da altura}

Inicialmente as medições de altura dos piquetes foram realizadas com a utilização de uma régua. Mediase do nível do solo até a curvatura da folha mais alta 
TABELA 1 - Dados climáticos considerados durante o período experimental (de Agosto/98 a Abril/99) e comparação com os dados dos últimos 80 anos.

\begin{tabular}{|c|c|c|c|c|c|c|}
\hline \multirow[b]{2}{*}{ Mês/ano } & \multicolumn{3}{|c|}{ Temperatura } & \multirow{2}{*}{$\frac{\text { Chuva }}{\text { Total mensal }}$} & \multirow{2}{*}{$\frac{\text { Umidade Relativa }}{\text { Média }}$} & \multirow{2}{*}{$\frac{\text { Insolação }}{\text { Média }}$} \\
\hline & Máxima média & Mínima média & Média & & & \\
\hline & - - & 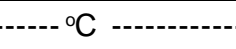 & - & $\mathrm{mm}$ & $\%$ & $\mathrm{~h} \mathrm{dia}^{-1}$ \\
\hline Agosto/98 & 27,4 & 14,4 & 20,9 & 21,8 & 77,0 & 6,2 \\
\hline Média (80 anos) & 27,0 & 10,8 & 18,9 & 29,5 & 61,7 & 7,9 \\
\hline Setembro/98 & 27,7 & 15,3 & 21,5 & 63,3 & 65,8 & 5,7 \\
\hline Média (80 anos) & 27,7 & 13,2 & 20,4 & 63,0 & 62,2 & 6,7 \\
\hline Outubro/98 & 27,1 & 16,3 & 21,7 & 183,1 & 83,4 & 5,4 \\
\hline Média (80 anos) & 28,6 & 15,4 & 22,0 & 110,4 & 68,8 & 6,7 \\
\hline Novembro/98 & 30,0 & 16,5 & 23,2 & 26,6 & 76,6 & 7,8 \\
\hline Média (80 anos) & 23,1 & 16,6 & 29,6 & 128,0 & 69,0 & 7,6 \\
\hline Dezembro/98 & 30,0 & 19,2 & 24,6 & 200,2 & 74,3 & 6,6 \\
\hline Média (80 anos) & 28,9 & 17,7 & 23,3 & 199,0 & 71,5 & 6,3 \\
\hline Janeiro/99 & 30,6 & 20,4 & 25,5 & 382,8 & 88,0 & 4,9 \\
\hline Média (81 anos) & 29,6 & 18,7 & 24,1 & 225,1 & 73,3 & 6,3 \\
\hline Fevereiro/99 & 30,7 & 20,1 & 25,4 & 198,3 & 90,0 & 5,4 \\
\hline Média (81 anos) & 30,2 & 19,0 & 24,6 & 188,0 & 75,5 & 6,4 \\
\hline Março/99 & 31,4 & 19,0 & 25,2 & 210,8 & 80,7 & 7,7 \\
\hline Média (81 anos) & 29,9 & 18,2 & 24,0 & 143,4 & 75,9 & 6,7 \\
\hline Abril/99 & 28,5 & 15,2 & 22,0 & 89,0 & 80,7 & 8,1 \\
\hline Média (81 anos) & 28,3 & 15,4 & 21,1 & 64,8 & 74,2 & 7,5 \\
\hline
\end{tabular}

Fonte: Departamento de Ciências Exatas - USP/ESALQ.

sem comprimi-la, em 18 pontos por piquete. Contudo, através desse método, o coeficiente de variação foi muito alto (40-50\%) e por isso, o método foi modificado. Passou-se a utilizar uma folha de acetato (transparência) colocada aleatoriamente em alguns pontos da pastagem, onde eram lidas a maior e a menor altura com uma régua, obtendo-se assim a média. A princípio foram medidos 18 pontos/piquete, mas, como o coeficiente de variação (CV) diminuiu sensivelmente (de 40 para 20\%) usou-se apenas 12 pontos/piquete. Contudo, apesar de expressar a altura eficientemente, o método necessitava de grande disponibilidade de tempo e pessoas, o que tornou-se limitante. A altura foi monitorada semanalmente através de 20 medições, em cada piquete, com o prato ascendente (Frame, 1981). Essa avaliação era feita duas vezes por semana, obtendo-se a altura comprimida. Uma vez por mês era feita a calibração da altura comprimida com a altura não comprimida (régua), sendo esta última a utilizada como referência para o monitoramento dos tratamentos. Determinada a altura do pasto na unidade experimental, eram adicionados ou retirados animais em função desta e das alturas medidas anteriormente. Os animais traçadores de ganho de peso permaneceram o tempo todo dentro da unidade experimental enquanto que ovinos adultos eram utilizados para a regular a carga e manter a altura desejada. Houve situações onde 0 acúmulo de matéria seca da unidade experimental era inferior ao necessário para o consumo dos traçadores. Nesses casos, os animais eram retirados com a finalidade de assegurar a manutenção das condições do pasto prérestabelecidas. Os animais marcadores eram colocados em pastagens extras onde continuavam recebendo 0 mesmo tipo de alimentação. Todos os animais que entravam ou saíam das unidades experimentais eram submetidos a jejum de 16 horas e pesados.

\section{Calibração: Altura x Massa}

Uma vez por mês foi feita a calibração entre altura e massa de forragem. Com anéis de $0,25 \mathrm{~m}^{2}$ de área, eram escolhidos dois pontos dentro de cada unidade experimental procurando sempre o ponto mais baixo e 0 ponto mais alto a fim de se obter a maior amplitude de alturas. Era medida a altura dentro do anel com a régua e com o prato ascendente e depois a forragem era cortada ao nível do solo, lavada para eliminação de resíduos de terra e fezes, seca em estufa a $65^{\circ} \mathrm{C}$ e pesada. Com isso, foi possível determinar uma regressão linear ou equação de calibração entre altura não comprimida e massa e outra entre altura comprimida e massa em cada mês. Essas equações foram utilizadas para os cálculos de acúmulo e densidade de forragem.

\section{Acúmulo de forragem}

$\mathrm{O}$ acúmulo de forragem foi obtido através do uso de gaiolas de exclusão $(0,70 \times 0,70 \times 0,70 \mathrm{~m})$. 
Foram utilizadas três gaiolas por unidade experimental, que, após um intervalo de 21 dias, eram retiradas e a altura era medida com o prato ascendente (5 leituras por gaiola). Essa altura era posteriormente transformada em massa através da equação de calibração. Após a medição, as gaiolas eram reancoradas em novos pontos representativos da condição media do pasto na unidades experimental.

O acúmulo de forragem foi estimado pelo método agronômico da diferença conforme a equação (Davies et al., 1993): $A F=M F_{f}-M F_{i}$, sendo: $A F=$ acúmulo de forragem; $\mathrm{M} \mathrm{F}_{f}=$ massa de forragem, sob a gaiola, no último dia de exclusão (dia 21); $\mathrm{M} \mathrm{F}_{\mathrm{i}}=$ massa de forragem média da unidade experimental por ocasião da colocação das gaiolas (dia 1).

\section{Densidade de forragem}

A densidade de forragem foi calculada a partir dos dados de altura média não comprimida do pasto e da massa de forragem correspondente, calculada a partir das equações de calibração. $O$ valor de massa de forragem foi dividido pela altura média e o quociente obtido foi a densidade.

\section{Disponibilidade de forragem}

A disponibilidade de forragem foi obtida através da relação entre a taxa de acúmulo de forragem e o total de peso vivo dos animais presentes na área no período correspondente, expressando disponibilidade em kg MS/ 100kg PV.dia.

\section{Composição química}

As amostras foram coletadas pelo método de simulação de pastejo segundo Sollenberger \& Cherney (1995). O material coletado foi acondicionado em saco plástico e levado para uma câmara fria. De cada amostra foi retirada uma subamostra para fins de separação morfológica. O restante da amostra era seco em estufa a $65^{\circ} \mathrm{C}$, por 48 horas. Após a secagem, o material era moído em moinho tipo Wiley com peneira de $1 \mathrm{~mm}$ e encaminhado para as análises químicas. $O$ valor nutritivo foi determinado através das determinações dos teores de matéria seca (MS) e matéria mineral (MM) de acordo com Silva (1990), proteína bruta (PB) pelo método da AOAC seguindo Pereira \& Rossi Júnior (1996), fibra em detergente neutro (FDN), fibra em detergente ácido (FDA) e lignina segundo o método de Van Soest et al. (1991) com o aparelho Fiber Analyser. A digestibilidade in vitro da matéria orgânica (DIVMO) foi obtida através da utilização do aparelho Daisy Incubator II com procedimento segundo Tilley e Terry (1966).

\section{Composição morfológica da forragem pastejada}

A subamostra gerada a partir das amostras utilizadas para análise química foi dividida em: folhas verdes folhas senescentes, pseudocaule (hastes + bainhas das folhas) e material morto. Cada componente foi colocado em saquinhos de papel seco por 48 horas e pesado. As proporções dos componentes nas amostras foram calculadas como a porcentagem do peso total.

\section{Estrutura do pasto}

A avaliação de estrutura do pasto foi feita através de amostragens destrutivas onde se fez manualmente a separação morfológica da forragem colhida em folhas (lâminas foliares), pseudocaules (hastes + bainhas) e material morto. Foram utilizados dois quadrados de 0,25 $\mathrm{m}$ de lado por unidade experimental, sendo que toda a forragem colhida no interior de cada quadrado foi cortada ao nível do solo.

\section{Desempenho Animal}

Para avaliar desempenho animal foram utilizados ovinos deslanados em crescimento, mestiços da raça Santa Inês com grau de sangue variável, com idade média de 9 meses no início do período experimental. Os animais traçadores foram substituídos quando completaram um ano de vida. Os novos animais tinham idade média de 4 meses e foram alocados às unidades experimentais em novembro de 1998. Os animais foram separados segundo raça, sexo, idade e peso seguindo essa ordem de importância. O pares traçadores somavam o mesmo peso para todas as unidades experimentais dentro do bloco. A distribuição dos grupos para os blocos e dos pares para as unidades experimentais foi aleatória.

Para avaliar ganho de peso vivo individual, foram utilizados dois animais traçadores por unidade experimental. Foram avaliados ganho de peso vivo (GPV) por animal e por área e capacidade de suporte. As pesagens foram mensais, sempre no mesmo horário do dia com jejum de água e alimento por 16 horas. Os animais que regulavam a taxa de lotação nas unidades experimentais para manutenção da altura de pastejo eram pesados em jejum, na entrada e na saída. Todos os animais foram vermifugados a uma frequência máxima de 30 dias, devido a alta reinfestação que ocorre em sistemas de pastejo com lotação contínua.

\section{Estimativas de consumo}

O consumo de forragem foi estimado através de cálculos feitos a partir do peso do animal, ganho de peso vivo, energia metabólica do alimento e a exigência nutricional da categoria animal, segundo Le Du \& Penning (1982). As equações utilizadas para esta estimativa foram as seguintes: $E D=(0,1233 \times \mathrm{PB})+(0,1705 \times \mathrm{DMO})+$ 0,285; $\mathrm{Ema}=0,815 \times \mathrm{ED} ; \mathrm{Emc}=\operatorname{antilog}[1,11 \times \log 10$ $(\mathrm{GPI})+(0,004 \times \mathrm{PV})-2,1] /(0,0435 \times \mathrm{Ema}) ; \mathrm{Emm}=1,4$ $+(0,15 \times \mathrm{PV})$ e Consumo $=(E \mathrm{Emc}+\mathrm{Emm}) /$ Ema Onde: $\mathrm{ED}$ - Energia Digestível, PB - Proteína Bruta; DMO Digestibilidade da Matéria Orgânica; Ema - Energia Metabolizável do alimento; Emc - Energia Metabolizável de Crescimento; GPI - Ganho de Peso Individual; PV - Peso Vivo e Emm - Energia Metabolizável de Manutenção.

A partir dessas equações foi possível obterem-se também valores de energia metabolizável da forragem "consumida". 


\section{RESULTADOS E DISCUSSÃO}

\section{Composição morfológica e Valor nutritivo}

O tratamento de $50 \mathrm{~mm}$ apresentou, em média, a maior porcentagem de folhas "pastejadas" (76\%), seguido do tratamento de $100 \mathrm{~mm}$ ( $71 \%$ ). Esses dois tratamentos também apresentaram as menores porcentagem de pseudohastes, em média, 13,8\% e 15,5\% para 50 e $100 \mathrm{~mm}$, respectivamente. Pastos mais altos apresentaram, em geral, maior porcentagem de pseudohastes e menor porcentagem de folhas. A participação de material morto e folhas senescentes foi pequena na primavera e tendeu a diminuir no verão. Isso pode estar relacionado com o fato de ter havido maior disponibilidade de forragem no verão, proporcionando maior oportunidade de seleção (TABELA 2 e 4).

A composição química da forragem "pastejada" revelou uma maior concentração de proteína bruta para as pastos mais baixos $(50 \mathrm{~mm})$, independente da estação do ano, cerca de $20 \%$ de PB (TABELAS 2 e 4). Vilela \& Alvim (1996) encontraram valores semelhantes (16 a $23 \%$ de PB) para Coastcross-1 pastejado por bovinos leiteiros. Valores de $15 \%$ de proteína foram encontrados por Hill et al. (1993) e 13\% foi encontrado por Pedreira (1995) em amostras de Tifton 85 pastejado por novilhas que exercem menor grau de seleção que ovinos (Hodgson, 1990). Gomide (1996) encontrou 16\% de PB nas folhas e $10 \%$ de PB nas pseudohastes, indicando que diferentes proporções dos componentes selecionados para ingestão determinam diferenças no valor nutritivo. Alvim et al. (1996) encontraram teores de PB maiores para a época das chuvas (primavera e verão) variando entre 13 e 19\% em relação a época seca (outono e inverno) que variou entre 11 e $16 \%$.

A maior participação de folhas na forragem selecionada para os pastos de $50 \mathrm{~mm}$ propiciou uma menor proporção de parede celular (FDN) para este tratamento $(64 \%)(P<0,05)$. Os menores valores de FDN (64 a $67 \%$ ) foram observados para os pastos mais baixos (50 e $100 \mathrm{~mm}$ ) quando comparados com os valores de 68 a $70 \%$ dos pastos mais altos (150 e 200 $\mathrm{mm})(\mathrm{P}<0,05)$. Alvim et al. (1996) encontraram teores de FDN variando entre 63 e $70 \%$ na época das chuvas e 64 e $74 \%$ na época seca para Coastcross. Vilela \& Alvim (1996) encontraram valores semelhantes variando entre 64 e $71 \%$ para Coastcross. Para Tifton 85, Pedreira (1995) encontrou valores próximos a $80 \%$ de FDN. Os valores de proteína bruta e FDN tenderam a aumentar durante o período de verão.

Os teores de fibra em detergente ácido seguiram a mesma tendência que os de FDN. Os pastos de $50 \mathrm{~mm}$ apresentaram proporções mais baixas de FDA (25\%) $(P<0,05)$ que os demais tratamentos $(29 \%)$ e tenderam a aumentar no período de verão. Hill et al. (1993) encontraram $73 \%$ de FDN e $34 \%$ de FDA na forragem pastejada por novilhos nas condições da Georgia.

TABELA 2 - Valor nutritivo e composição morfológica das pastagens de Tifton 85 mantidas sob quatro condições de pasto durante o período da primavera da estação de crescimento de 1998/1999.

\begin{tabular}{|c|c|c|c|c|c|}
\hline \multirow[b]{2}{*}{ Primavera } & \multicolumn{4}{|c|}{ Altura (mm) } & \multirow[b]{2}{*}{ EPM } \\
\hline & 50 & 100 & 150 & 200 & \\
\hline \multicolumn{6}{|l|}{ Valor nutritivo } \\
\hline Proteína bruta (\%) & 19,9 a & $16,9 a b$ & $14,9 a b$ & $15,0 \mathrm{~b}$ & 1,16 \\
\hline FDN $(\%)$ & $63,9 \mathrm{~b}$ & $66,2 \mathrm{ab}$ & 66,9 a & 67,7 a & 0,84 \\
\hline FDA (\%) & $25,5 \mathrm{~b}$ & $26,9 a b$ & $27,7 a b$ & $28,5 a b$ & 0,54 \\
\hline Lignina (\%) & 6,8 a & 7,3 a & 7,4 a & 8,3 a & 1,15 \\
\hline DIVMO (\%) & 82,9 a & $79,4 a b$ & $77,2 \mathrm{~b}$ & $77,0 \mathrm{~b}$ & 1,14 \\
\hline \multicolumn{6}{|c|}{ Composição da forragem pastejada } \\
\hline Folhas (\%) & 76,8 a & $73,4 a b$ & $66,6 \mathrm{~b}$ & $68,4 a b$ & 2,95 \\
\hline Pseudohastes (\%) & $12,1 \mathrm{~b}$ & $13,2 \mathrm{ab}$ & $14,6 a b$ & $16,6 \mathrm{a}$ & 1,90 \\
\hline Material Morto (\%) & $8,0 \mathrm{~b}$ & $11,1 a b$ & $12,8 a b$ & $14,4 \mathrm{a}$ & 1,89 \\
\hline Folhas Senescentes (\%) & $3,1 \mathrm{a}$ & $2,3 \mathrm{a}$ & $4,3 \mathrm{a}$ & $4,7 \mathrm{a}$ & 0,98 \\
\hline \multicolumn{6}{|l|}{ Estrutura da planta } \\
\hline Folhas (\%) & $17,4 a b$ & $17,6 a b$ & $15,3 \mathrm{~b}$ & $18,9 \mathrm{a}$ & 1,04 \\
\hline Pseudohastes (\%) & $50,1 \mathrm{a}$ & $44,9 \mathrm{a}$ & $41,8 \mathrm{a}$ & $42,9 \mathrm{a}$ & 1,96 \\
\hline Material morto (\%) & $32,5 \mathrm{~b}$ & $37,6 a b$ & $43,0 \mathrm{a}$ & $38,3 a b$ & 2,26 \\
\hline $\begin{array}{l}\text { Relação } \\
\text { pseudohaste/folhas }\end{array}$ & $3,2 \mathrm{a}$ & $3,3 \mathrm{a}$ & $3,2 \mathrm{a}$ & $2,7 \mathrm{a}$ & 0,38 \\
\hline $\begin{array}{l}\text { Densidade de forragem } \\
\left(\mathrm{kg} \mathrm{MS} \mathrm{cm}^{-1} \mathrm{ha}^{-1}\right)\end{array}$ & $607 \mathrm{a}$ & $480 \mathrm{~b}$ & $422 \mathrm{c}$ & $369 d$ & 13,2 \\
\hline
\end{tabular}

Médias seguidas de letras diferentes na mesma linha são diferentes $(P<0,05)$.

EPM- Erro Padrão da Média. 
Os teores de lignina não apresentaram diferenças entre as alturas $(P>0,05)$. Contudo, no verão (3 a 4\%) os valores foram consideravelmente menores que na primavera (7 a $8 \%$ ).

A DIVMO apresentou valores elevados para todos os tratamentos ( $>75 \%$ ) com os maiores valores observados para os pastos mais baixos ( 50 e $100 \mathrm{~mm}$ ), ao redor de $84 \%(\mathrm{P}<0,05)$. Somente $60 \%$ de DIVMS foi observado por Hill et al. (1993), isso devido ao menor teor de proteína e maiores proporções de fibra presentes na dieta selecionada pelo bovino. Vilela \& Alvim (1996) encontraram valores de 61 a $66 \%$ de DIVMS para Coastcross.

Os teores de proteína e DIVMO podem estar relacionados com a proporção de folhas na amostra "consumida". Pode-se observar que as três variáveis seguem a mesma tendência para todas as alturas (TABELAS 2 e 4).

A partir dos teores de proteína e digestibilidade foi calculada a concentração de Energia Metabolizável da forragem. Os valores obtidos variaram de 10 a 14,5 MJ/ $\mathrm{kg}$ MS. Valores de 10 a $11 \mathrm{MJ} / \mathrm{kg}$ MS são relatados para azevém perene e são considerados elevados (Le Du \& Penning, 1982). Pastos mais altos apresentaram menores concentrações de energia metabolizável $(P<0,05)$.

Pela composição descrita da forragem "pastejada", pastagens de Tifton 85 não apresentaram limitações no valor nutritivo para interferir no consumo de forragem. Contudo, aspectos estruturais e quantidade de alimento disponível no pasto também interferem no consumo de maneira decisiva (Hodgson, 1990).

TABELA 3 - Respostas animais e das pastagens de Tifton 85 mantidas sob quatro condições de pasto durante o período da primavera da estação de crescimento de 1998/1999.

\begin{tabular}{|c|c|c|c|c|c|}
\hline \multirow[b]{2}{*}{ Primavera } & \multicolumn{4}{|c|}{ Altura (mm) } & \multirow[b]{2}{*}{ EPM } \\
\hline & 50 & 100 & 150 & 200 & \\
\hline \multicolumn{6}{|l|}{ Respostas da pastagem e dos animais } \\
\hline Taxa de acúmulo (kg MS ha-1 dia $\left.^{-1}\right)$ & 65,0 a & $62,0 \mathrm{a}$ & 67,0 a & $86,0 \mathrm{a}$ & 11,28 \\
\hline Capacidade de carga (kg PV ha-1 dia $^{-1}$ ) & 2550 a & $1880 \mathrm{~b}$ & $1320 \mathrm{~b}$ & $1420 \mathrm{~b}$ & 188,92 \\
\hline Disponibilidade de forragem (\% PV) & $2,4 \mathrm{c}$ & $3,6 \mathrm{bc}$ & $5,3 a b$ & 6,6 a & 0,64 \\
\hline Ganho por animal (g/animal.dia) & $-20,8 c$ & $12,5 \mathrm{~b}$ & $17,3 \mathrm{~b}$ & $35,3 \mathrm{a}$ & 3,76 \\
\hline Ganho por área $\left(\mathrm{kg} \mathrm{ha}^{-1} \mathrm{dia}^{-1}\right)$ & $-3,0 b$ & $1,3 a b$ & $1,8 \mathrm{a}$ & $1,8 \mathrm{a}$ & 1,01 \\
\hline Consumo de forragem (g MS/animal.dia) & $37,5 \mathrm{~b}$ & $372,5 \mathrm{a}$ & 325,0 a & $471,5 \mathrm{a}$ & 43,23 \\
\hline
\end{tabular}

Médias seguidas de letras diferentes na mesma linha são diferentes $(P<0,05)$.

EPM- Erro Padrão da Média.

TABELA 4 - Valor nutritivo e composição morfológica de pastagens de Tifton 85 mantidas sob quatro condições de pasto durante o período do verão da estação de crescimento de 1998/1999.

\begin{tabular}{|c|c|c|c|c|c|}
\hline \multirow[b]{2}{*}{ Verão } & \multicolumn{4}{|c|}{ Altura $(\mathrm{mm})$} & \multirow[b]{2}{*}{ EPM } \\
\hline & 50 & 100 & 150 & 200 & \\
\hline \multicolumn{6}{|l|}{ Valor nutritivo } \\
\hline Proteína bruta (\%) & $21,3 \mathrm{a}$ & $18 a b$ & $15,9 \mathrm{~b}$ & $15,6 \mathrm{~b}$ & 0,82 \\
\hline FDN (\%) & $64,4 \mathrm{c}$ & $67,9 \mathrm{~b}$ & $69,3 \mathrm{~b}$ & 70,0 a & 0,72 \\
\hline FDA (\%) & $25,6 \mathrm{~b}$ & $28,0 \mathrm{a}$ & 29,3 a & $29,4 a$ & 0,42 \\
\hline Lignina (\%) & $4,1 \mathrm{a}$ & $4,3 \mathrm{a}$ & 4,6 a & $3,8 \mathrm{a}$ & 0,46 \\
\hline DIVMO (\%) & $85,2 \mathrm{a}$ & $80,7 \mathrm{~b}$ & $76,2 \mathrm{c}$ & $78,0 \mathrm{bc}$ & 1,11 \\
\hline \multicolumn{6}{|l|}{ Composição da forragem pastejada } \\
\hline Folhas (\%) & $75,1 \mathrm{a}$ & $66,0 \mathrm{~b}$ & $64,1 \mathrm{~b}$ & $69,9 a b$ & 2,61 \\
\hline Pseudohastes (\%) & $15,0 \mathrm{~b}$ & $18,7 \mathrm{ab}$ & $20,2 \mathrm{a}$ & 19,8 a & 2,01 \\
\hline Material Morto (\%) & $8,1 \mathrm{a}$ & 11,7 a & $12,0 \mathrm{a}$ & $7,6 \mathrm{a}$ & 1,48 \\
\hline Folhas Senescentes(\%) & $1,8 \mathrm{a}$ & $3,6 \mathrm{a}$ & $3,7 \mathrm{a}$ & $2,7 \mathrm{a}$ & 0,73 \\
\hline \multicolumn{6}{|l|}{ Estrutura da planta } \\
\hline Folhas(\%) & $19,4 \mathrm{a}$ & $19,2 \mathrm{a}$ & $16,5 \mathrm{a}$ & $15,4 \mathrm{~b}$ & 1,05 \\
\hline Pseudohastes(\%) & 51,8 a & 46,7 a & $45,0 \mathrm{a}$ & $47,2 \mathrm{a}$ & 2,23 \\
\hline Material morto(\%) & 28,8 a & $34,1 a b$ & $38,5 \mathrm{~b}$ & $37,4 \mathrm{~b}$ & 2,49 \\
\hline Relação pseudohaste/folhas & $2,8 \mathrm{a}$ & $2,8 \mathrm{a}$ & $3,0 \mathrm{a}$ & $3,3 \mathrm{a}$ & 0,26 \\
\hline Densidade de forragem(kg MS $\left.\mathrm{cm}^{-1} \mathrm{ha}^{-1}\right)$ & $424 \mathrm{a}$ & $356 \mathrm{~b}$ & $329 \mathrm{c}$ & $317 d$ & 1,78 \\
\hline
\end{tabular}

Médias seguidas de letras diferentes na mesma linha são diferentes $(P<0,05)$.

EPM- Erro Padrão da Média. 


\section{Densidade de forragem e Estrutura do pasto}

Entre os principais fatores estruturais está a densidade de forragem. Os valores seguem uma tendência uniforme dentro das estações, ou seja, densidades mais altas para pastos mais baixos. A densidade da forragem diminuiu da primavera para o verão independente da altura. Isso foi consistente com as variações das proporções de material morto dos pastos. Durante a primavera ou verão, os maiores valores de densidade foram para os pastos de $50 \mathrm{~mm}$ seguido por 100,150 e $200 \mathrm{~mm}(P<0,05)$.

Pastos mais baixos apresentaram menores proporções de material morto tanto na primavera como no verão, com médias de verão sendo inferiores às médias de primavera. Esse fato, provavelmente, foi resultado da melhoria das condições de decomposição de matéria orgânica no verão (elevação da temperatura e da quantidade de chuvas).

A proporção de folhas no perfil total do pasto, não apresentou uma tendência constante, pois está muito relacionada à velocidade de crescimento e à lotação resultante das alturas estabelecidas, dois fatores que atuam simultaneamente. Contudo, pode-se salientar a grande seletividade por folhas exercida pelos animais dentro do perfil da pastagem. Em pastos com cerca de 15 a $20 \%$ de folhas, a dieta selecionada continha 64 a $77 \%$ de folhas. Gomide (1996) encontrou 49\% de folhas para áreas de Tifton 85 cortadas a cada 28 dias. A proporção de folhas variou pouco durante a primavera nos pastos de $200 \mathrm{~mm}$, os quais apresentaram os menores valores durante o período de verão (TABELAS 2 e 4).

A porcentagem de folhas pastejadas apresentou a mesma tendência que a porcentagem de folhas do perfil do pasto. As proporções dos outros componentes do pasto mostraram o mesmo comportamento, indicando uma possível dependência da composição da dieta em relação à estrutura do pasto apresentada ao animal. A porcentagem de pseudohastes foi alta para todas as alturas, apresentando valores de 30 a $60 \%$ e proporcionando uma dieta com 12 a $20 \%$ de pseudohastes. Nenhuma diferença foi observada nos dois períodos do ano para a \% de pseudohastes $(\mathrm{P}<0,05)$, o mesmo acontecendo para relação pseudohaste:folha (TABELAS 2 e 4).

\section{Respostas Animal e das Pastagens}

As taxas de acúmulo de matéria seca variaram de 65 a $86 \mathrm{~kg} \mathrm{MS} \mathrm{ha}^{-1} \mathrm{dia}^{-1}$ na primavera e 90 a $115 \mathrm{~kg} \mathrm{MS}$ $\mathrm{ha}^{-1} \mathrm{dia}^{-1}$ no verão (TABELAS 3 e 5 ). Valores da ordem de 30 a $90 \mathrm{~kg} \mathrm{MS} \mathrm{ha}^{-1} \mathrm{dia}^{-1}$ foram obtidos por Carnevalli \& da Silva (1999) para Coastcross-1.

Nenhuma diferença $(P>0,05)$ foi observada entre tratamentos dentro dos períodos considerados. Isso pode estar relacionado a técnica de gaiolas de exclusão utilizada, onde o acúmulo de forragem é provavelmente superestimado para os pastos mais baixos e sub-estimado para os pastos mais altos (Frame, 1981). Pastos mantidos continuamente baixos apresentam uma alta quantidade de perfilhos pequenos e uma baixa interceptação luminosa. Quando isolados da ação do animal, os perfilhos crescem em tamanho sem, contudo, haver uma redução proporcional na densidade de perfilhos durante o período de avaliação, ou seja, há um intervalo de tempo entre o sinal de compensação e a morte dos perfilhos para um novo equilíbrio. Durante esse período, portanto, estimativas de acúmulo são inflacionadas. Nos pastos mais altos, a interceptação de luz encontra-se mais próxima de valores elevados, diferentemente de pastos mais baixos. Assim, para o mesmo período de acúmulo (21 dias) chegase a interceptação de $95 \%$ de luz mais cedo com redução drástica do acúmulo no período. As taxas de acúmulo no verão foram cerca de $30 \%$ maiores que as taxas na primavera, aumento este proporcionado pela maior disponibilidade de fatores de crescimento (TABELA 1).

A produção total de forragem obtida no período experimental (9 meses) para cada altura foi de 17.820 , $17.560,17.590$ e $21.520 \mathrm{~kg} \mathrm{MS} \mathrm{ha}^{-1}$ para 50, 100, $150 \mathrm{e}$ $200 \mathrm{~mm}$, respectivamente. Vilela \& Alvim (1996) obtiveram $20 \mathrm{t} \mathrm{MS} / \mathrm{ha}$.ano para Coastcross e aproximadamente $23 \mathrm{t} / \mathrm{ha}$.ano foram estimados por

TABELA 5 - Respostas animais e das pastagens de Tifton 85 mantidas sob quatro condições de pasto durante o período do verão da estação de crescimento de 1998/1999.

\begin{tabular}{|c|c|c|c|c|c|}
\hline \multirow[b]{2}{*}{ Verão } & \multicolumn{4}{|c|}{ Altura $(\mathrm{mm})$} & \multirow[b]{2}{*}{ EPM } \\
\hline & 50 & 100 & 150 & 200 & \\
\hline \multicolumn{6}{|l|}{ Respostas da pastagem e dos animais } \\
\hline Taxa de acúmulo (kg MS ha-1 dia $\left.^{-1}\right)$ & $100,0 \mathrm{a}$ & $100,0 \mathrm{a}$ & $90,0 \mathrm{a}$ & $113,0 \mathrm{a}$ & 10,62 \\
\hline Capacidade de carga (kg PV ha-1 dia $^{-1}$ ) & 2520 a & $1790 \mathrm{~b}$ & $1860 \mathrm{~b}$ & $1800 \mathrm{~b}$ & 165,99 \\
\hline Disponibilidade de forragem (\% PV) & $4,0 a b$ & $7,1 \mathrm{a}$ & $5,0 a b$ & $6,5 a b$ & 0,86 \\
\hline Ganho por animal (g/animal.dia) & $31,3 \mathrm{a}$ & $35,5 \mathrm{a}$ & $46,8 \mathrm{a}$ & 44,8 a & 8,35 \\
\hline Ganho por área $\left(\mathrm{kg} \mathrm{ha}^{-1} \mathrm{dia}^{-1}\right)$ & $4,3 \mathrm{a}$ & $2,3 \mathrm{a}$ & $4,3 \mathrm{a}$ & $3,5 \mathrm{a}$ & 1,5 \\
\hline Consumo de forragem (g MS/animal.dia) & $317,5 \mathrm{~b}$ & $367,5 a b$ & 455,0 a & 440,0 a & 42,58 \\
\hline
\end{tabular}

Médias seguidas de letras diferentes na mesma linha são diferentes $(P<0,05)$.

EPM- Erro Padrão da Média. 
Carnevalli \& da Silva (1999) para o mesmo cultivar. Para Tifton 85, Hill et al. (1993) obtiveram aproximadamente 25 t MS ha $^{-1}$ ano $^{-1}$, com 170 dias de pastejo. Gomide (1996) obteve produção de $2.256 \mathrm{~kg} \mathrm{MS} /$ ha.corte de folhas e $1.552 \mathrm{~kg} \mathrm{MS} /$ ha.corte de pseudohastes.

Como consequência das taxas de acúmulo, a disponibilidade de forragem durante 0 verão foi superior a disponibilidade na primavera (2 a $6 \%$ na primavera e 4 a $7 \%$ no verão), particularmente para animais nos pastos de $50 \mathrm{~mm}$ (2 e 4\% para primavera e verão, respectivamente) (TABELAS 3 e 5). Pedreira (1995) obteve disponibilidades de forragem para Tifton 85 variando entre 0,6 a $1,4 \%$ do PV. A disponibilidade de forragem associada a uma capacidade de suporte semelhante entre primavera e verão $(P<0,05)$ e a composição química da forragem consumida, promoveu um aumento no ganho de peso por animal e por hectare da primavera para o verão. Este aumento foi mais expressivo para os pastos de $50 \mathrm{~mm}$. Para essa altura a capacidade de suporte foi de $2.500 \mathrm{~kg} \mathrm{PV} \mathrm{ha}^{-1} \mathrm{dia}^{-1}$. As demais alturas tiveram uma capacidade de suporte variando entre 1.300 e $1.800 \mathrm{~kg} \mathrm{PV} \mathrm{ha}^{-1} \mathrm{dia}^{-1}$ (TABELAS 3 e 5). Hill et al. (1993) obtiveram uma carga animal de $1.320 \mathrm{~kg} \mathrm{PV}$ $\mathrm{ha}^{-1} \mathrm{dia}^{-1}$ com um ganho de peso individual de $0,650 \mathrm{~kg} /$ animal.dia e $790 \mathrm{~kg} \mathrm{ha}^{-1} \mathrm{ano}^{-1}$, para novilhos de corte em crescimento em pastagens de Tifton 85. Pedreira (1995) obteve uma carga animal anual variando entre $1.400 \mathrm{e}$ $2.100 \mathrm{~kg} \mathrm{PV} \mathrm{ha}^{-1} \mathrm{dia}^{-1}$ e um ganho de peso de 0,400 a 0,700 $\mathrm{kg} \mathrm{PV} \mathrm{ha-1}$ dia $^{-1}$ para novilhas. Mislevy \& Pate (1996) obtiveram ganhos com bovinos da ordem de $0,380 \mathrm{~kg} /$ animal.dia e $495 \mathrm{~kg} \mathrm{ha}^{-1}$ ano $^{-1}$ para Florakirk.

As estimativas de consumo de forragem revelaram que 0 aumento no desempenho animal foi uma possível consequência do maior consumo de forragem, provavelmente devido à oferta mais generosa observada durante o verão. Valores superiores de ganho de peso por animal e por hectare foram obtidos para os pastos mais altos (150 e $200 \mathrm{~mm}$ ) independente da estação do ano (TABELAS 3 e 5).

Na TABELA 6 é apresentado o número de dias de pastejo no período total de experimento para cada altura. Não houve diferença $(P>0,05)$ para essa variável, apesar da necessidade de retirada dos animais das unidades experimentais ter sido mais frequente nos pastos de 100 e $200 \mathrm{~mm}$.

TABELA 6 - Número de dias de pastejo de Tifton 85 nas quatro intensidades de pastejo $(50,100,150 \mathrm{e}$ 200).

\begin{tabular}{cccccc}
\hline & \multicolumn{4}{c}{ Altura $(\mathrm{mm})$} \\
\cline { 2 - 5 } & 50 & 100 & 150 & 200 & EPM \\
\hline Dias de pastejo & 258 a & 230 a & 248 a & 235 a & 4,6
\end{tabular}

Médias seguidas de letras diferentes na mesma linha são diferentes $(P<0,05)$.

EPM - Erro Padrão da Média

\section{CONCLUSÕES}

- O valor nutritivo da forragem consumida de Tifton 85 foi compatível com ganhos de peso elevados (PB $=15$ a $21 \%$ e DIVMO = 77 e $85 \%$ ).

- O ganho de peso por animal e por área foi fortemente influenciado pela disponibilidade diária de forragem e pela capacidade de carga dos pastos.

- Os maiores ganhos de peso por animal e por área foram obtidos com disponibilidades de forragem de 5 a $6 \%$ PV.

- A estrutura do pasto e sua composição morfológica foram afetados pelo manejo da pastagem, particularmente com referência à proporção de material morto;

- A escolha do melhor regime de desfolha dependerá dos objetivos pré-estabelecidos para a produção animal e o nível de eficiência desejado de utilização do pasto, havendo a necessidade de cautela no uso de pastagens de $50 \mathrm{~mm}$ quando a taxa de acúmulo de matéria seca for baixa.

\section{AGRADECIMENTOS}

Ao GEPF (Grupo de Estudos de Plantas Forrageiras) pelo auxílio nos trabalhos de campo. À FAPESP e à CAPES pelo financiamento do projeto.

\section{REFERÊNCIAS BIBLIOGRÁFICAS}

ALVIM, M.J.; BOTREL, M.A.; PASSOS, L.P.; BRESSAN, M.; VILELA, D. Efeito da frequência de cortes e do nível de nitrogênio sobre a produção e qualidade da matéria seca do Coastcross. In: WORKSHOP SOBRE O POTENCIAL FORRAGEIRO DO GÊNERO CYNODON, Juiz de Fora, 1996. Anais. Juiz de Fora: EMBRAPA, CNPGL, 1996. p. 45-56.

CARNEVALLI, R.A.; DA SILVA, S.C. Validação de técnicas experimentais para avaliação de características agronômicas e ecológicas de pastagens de Cynodon dactylon cv. Coastcross-1. Scientia Agricola, v.56, p.489-499, 1999.

DAVIES, D.A.; FUTHERGILL, M.; MORGAM, C.T. Assessment of constrasting perenial ryegrasses with white clover, under continuous stocking in the uplands. 5 - Herbage production, quality and intake in years 4-6. Grass and Forage Science, v.48, p.213-222, 1993.

FRAME, J. Herbage mass. In: HODGSON, J.; BAKER, R.D.; DAVIES, A.; LAIDLAW, A.S.; LEAVER, J.D. (Ed.) Sward meansurement handbook. Hurley: Bristish Grassland Society, 1981. p.39-67.

GOMIDE, C.C.C. Algumas características fisiológicas e químicas de cinco cultivares de Cynodon. Jaboticabal, 1996. 77p. dissertação (Mestrado) - Faculdade de Ciências Agrárias e Veterinárias, Universidade Estadual Paulista Júlio de Mesquita Filho.

HILL, G.M.; GATES, R.N.; BURTON, G.W. Forage quality and grazing steer performance from "Tifton 85"and "Tifton-78" bermudagrasses pastures. Journal of Animal Science, v.71, p.3219-3225, 1993.

HODGSON, J. Swards conditions, herbage allowance and animal production: na evaluation of research results. Proceedings of New Zealand Society of Animal Production, v.44, p.99-104, 1984.

HODGSON, J. Grazing management: science into pratice. New York: John Wiley \& Sons., 1990. 203p. 
MISLEVY, P.; PATE, F.M. Establishment, management and utilization of Cynodon grasses in Florida. In: WORKSHOP SOBRE O POTENCIAL FORRAGEIRO DO GÊNERO CYNODON, Juiz de Fora, 1996. Anais. Juiz de Fora : EMBRAPA,CNPGL, 1996. p.127-138.

LE DU, Y.L.P.; PENNING, P.D. Animal based tecniques for estimating herbage intake. In: LEAVER, J.D. Herbage intake handbook. Hurley: British Grassland Society, 1982, p.37-76.

PARSONS A.J.; LEAFE, E.L.; COLLET B.; PENNING, P.D.; LEWIS, J. The physiology of grass production under grazing. 2. Photosynthesis, crop growth and animal intakeof continuously, grazed pastures. Journal of Applied Ecology, v.20, p.127-139, 1983.

PENNING, P.D.; PARSONS, A.J.; ORR, R.J.; TREACHER, T.T. Intake and behaviour responses by sheep to changes in sward characteristics under continous stocking. Grass and Forage Science, v.46, p.15-28, 1991.

PEDREIRA, C.G.S. Plant and animal responses on grazed pastures of 'Florakirk' and 'Tifton 85 ' bermudagrasses. Florida, 1995. 153p. Thesis (Ph.D.) - University of Florida.

PEREIRA, J.R.A.; ROSSI JR, P. Manual prático de avaliação nutricional de alimentos. Piracicaba: FEALQ, 1996.
SILVA, D.J. Análise de alimentos: métodos químicos e biológicos. Viçosa: UFV, 1990.

SOLLENBERGER, L.E.; CHERNEY, D.J.R. Evaluating forage production and quality. The Science of Grassland Agriculture. Ames: lowa State University Press, p. 97-110, 1995.

TILLEY, J.M.A.; TERRY, R.A. A two-stage technique for the in vitro digestion of forage digestibility. Journal Britain Grassland Society 18:104-111, 1963.

VAN SOEST, P.J.; ROBERTSON, J.B.; LEWIS, B.A. Methods for dietary fiber, neutral detergent fiber and nonstarch polysaccharides in relation to animal nutrition. Journal of Dairy Science, v.74, p.3583-3597, 1991.

VILELA, D.; ALVIM, M.J.. Produção de leite em pastagem de Cynodon dactylon cv. Coastcross.. In: WORKSHOP SOBRE O POTENCIAL FORRAGEIRO DO GÊNERO CYNODON, Juiz de Fora, 1996. Anais. Juiz de Fora: EMBRAPA, CNPGL, 1996. p.77-92.

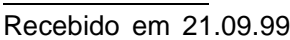

